\title{
Management of systemic lupus erythematosus during pregnancy: challenges and solutions
}

\author{
Caroline L Knight \\ Catherine Nelson-Piercy \\ Division of Women's Health, Women's \\ Health Academic Centre, King's \\ College London and King's Health \\ Partners, St Thomas' Hospital, \\ London, UK
}

This article was published in the following Dove Press journal: Open Access Rheumatology: Research and Reviews

10 March 2017

Number of times this article has been viewed
Correspondence: Catherine Nelson-Piercy

Division of Women's Health, Women's Health Academic Centre, King's College London and King's Health Partners, St Thomas' Hospital, Westminster Bridge Road, London SEI 7EH, UK Tel +442071887905

Email catherine.nelson-piercy@gstt.nhs.uk
Abstract: Systemic lupus erythematosus (SLE) is a chronic, multisystem autoimmune disease predominantly affecting women, particularly those of childbearing age. SLE provides challenges in the prepregnancy, antenatal, intrapartum, and postpartum periods for these women, and for the medical, obstetric, and midwifery teams who provide their care. As with many medical conditions in pregnancy, the best maternal and fetal-neonatal outcomes are obtained with a planned pregnancy and a cohesive multidisciplinary approach. Effective prepregnancy risk assessment and counseling includes exploration of factors for poor pregnancy outcome, discussion of risks, and appropriate planning for pregnancy, with consideration of discussion of relative contraindications to pregnancy. In pregnancy, early referral for hospital-coordinated care, involvement of obstetricians and rheumatologists (and other specialists as required), an individual management plan, regular reviews, and early recognition of flares and complications are all important. Women are at risk of lupus flares, worsening renal impairment, onset of or worsening hypertension, preeclampsia, and/or venous thromboembolism, and miscarriage, intrauterine growth restriction, preterm delivery, and/or neonatal lupus syndrome (congenital heart block or neonatal lupus erythematosus). A cesarean section may be required in certain obstetric contexts (such as urgent preterm delivery for maternal and/or fetal well-being), but vaginal birth should be the aim for the majority of women. Postnatally, an ongoing individual management plan remains important, with neonatal management where necessary and rheumatology followup. This article explores the challenges at each stage of pregnancy, discusses the effect of SLE on pregnancy and vice versa, and reviews antirheumatic medications with the latest guidance about their use and safety in pregnancy. Such information is required to effectively and safely manage each stage of pregnancy in women with SLE.

Keywords: systemic lupus erythematosus, preconception counseling, medication, management of pregnancy, pregnancy complications, neonatal lupus

\section{Introduction}

Systemic lupus erythematosus (SLE) is a rare, multisystem, chronic autoimmune disease which can vary from mild to life-threatening. ${ }^{1}$ It can present with a variety of symptoms including rash, arthritis, anemia, thrombocytopenia, serositis, nephritis, seizures, and/or psychosis. ${ }^{2}$ It typically shows a waxing and waning clinical course, but some patients have continuous disease activity. ${ }^{3}$

The overall incidence (newly diagnosed cases) in the UK in 1999-2012 was 4.9/100,000 person-years. The incidence of SLE in women is six times higher than in men in the UK (8.34 vs $1.44 / 100,000$ person-years); ${ }^{1}$ in premenopausal adults, the female-to-male ratio is $15: 1 .^{4}$ There are also marked variations in incidence in 
different ethnic groups. In the UK, the highest incidence is in Black Caribbean women (31.5/100,000 person-years), and the incidence in Black other $(22.3 / 100,000)$ and Black African (13.8/100,000) women is also increased. The rates in Pakistani (10.0/100,000), Indian (9.9/100,000), and Chinese women $(9.4 / 100,000)$ are lower with the lowest incidence in White women $(6.7 / 100,000){ }^{1}$

The overall prevalence (proportion of cases within a population) in the UK in 1999-2012 was 97/100,000. The rate was 6.8 times higher in women $(170 / 100,000)$. The prevalence was the highest in Black Caribbean patients $(518 / 100,000)$ and the lowest in White patients $(135 / 100,000) .{ }^{1}$ These are similar to the US: Black vs White $400 / 100,000$ vs $100 / 100,000$; female-to-male ratio is $10-15: 1 .^{3}$

Patients with SLE have increased mortality due to lupus complications or infection in earlier adult life, and myocardial infarction or stroke in later adult life. ${ }^{5}$ The overall survival in patients diagnosed with SLE is $92 \%$ after 10 years $;{ }^{6}$ thus, with modern drug therapies and management, many women with SLE of childbearing age are now conceiving. SLE is the commonest autoimmune rheumatic disease encountered in pregnancy; knowledge of pregnancy management in such patients is thus important.

\section{General principles}

Complications during pregnancy may be maternal (lupus flares, worsening renal impairment, onset of or worsening hypertension, development of preeclampsia, or venous thromboembolism [VTE]) and/or fetal-neonatal (miscarriage, intrauterine growth restriction [IUGR], preterm delivery, neonatal lupus syndrome [NLS]). ${ }^{7}$

As with many medical conditions in pregnancy, the best maternal and fetal-neonatal outcomes are obtained with a cohesive multidisciplinary approach. For patients with SLE, the multidisciplinary team may include a rheumatologist (ideally familiar with pregnancy in patients with SLE), obstetrician, nephrologist, fetal cardiologist, fetal medicine specialist, neonatologist, and/or specialist midwife. The woman's care should include effective prepregnancy risk assessment and stratification followed by individually tailored prepregnancy counseling. When she conceives, she should book early for pregnancy care with rheumatology and obstetric appointments in the first trimester and an individually tailored antenatal management plan. Early recognition and management of flares and complications (medical and/or obstetric) are important, with involvement of practitioners experienced in managing pregnancy in patients with SLE. Following delivery, an individually tailored postnatal management plan is equally important, including neonatal management where necessary and rheumatology follow-up.

This article examines challenges in the prepregnancy, antenatal, intrapartum, and postpartum periods for women with SLE and the medical, obstetric, and midwifery teams who provide their care.

\section{Prepregnancy challenge: stratifying individual patient risk}

The prepregnancy consultation should gather detailed information (Table 1) to deduce a woman's individual risks related to pregnancy. This should include past and current SLE disease activity (including most recent flare and frequency), preexisting organ damage (particularly cardiac, lung, and/or renal), medication history, and a recent serological profile (anti-dsDNA, anti-Ro/La antibodies, antiphospholipid antibodies [aPL], complement). The presence of any additional medical disorders should be elicited, in particular hypertension, diabetes, renal disease, and VTE, along with any additional medications. The past obstetric history should include gestation and outcome of all pregnancies, with particular reference to fetal and/or neonatal losses or complications (miscarriage, stillbirth, small-for-gestational age [SGA], preterm birth, congenital heart block [CHB], and/or the rash of neonatal lupus erythematosus [NLE]) and maternal complications (preeclampsia, antenatal or postpartum flares, VTE). Baseline blood pressure and urinalysis should be performed in clinic. Baseline blood tests (full blood count [FBC], urea and electrolytes [U\&E], creatinine, liver function tests [LFTs]) and serological profile, if not recently available, should be requested. ${ }^{8}$ Additional organ-specific tests (cardiac echo, lung function tests) may be helpful in assessing function in affected individuals.

Based on this information, women with SLE can be stratified into the following groups: 1) current remission, or stable low disease activity, with stable treatment; 2) early-stage or

Table I Prepregnancy consultation: information gathering

Past and current disease activity

Preexisting organ damage

Serological profile

Medication history

Additional medical disorders

Past obstetric history

Baseline blood pressure, urinalysis

FBC, U\&E, creatinine, LFTs \pm organ-specific investigations

Note: Data from Ateka-Barrutia et al. ${ }^{8}$

Abbreviations: FBC, full blood count; U\&E, urea and electrolytes; LFTs, liver function tests. 
Table 2 Relative contraindications to pregnancy

Severe lupus flare (including renal flare) within the past 6 months Stroke within the past 6 months

Pulmonary hypertension

Moderate-to-severe heart failure

Severe valvulopathy

Severe restrictive lung disease

Chronic kidney disease stage 4-5

Uncontrolled hypertension

Previous severe early-onset ( $<28$ weeks) preeclampsia or HELLP

syndrome despite therapy with aspirin plus heparin

Note: Data from $7,8,10,11$.

Abbreviation: HELLP Haemolysis, Elevated Liver enzymes, Low Platelet count.

currently active disease; or 3) severe impairment of organ function \pm preexisting severe organ damage. ${ }^{9}$

Women in group 3 are at particularly high risk of complications including worsening disease progression and end-organ failure, and there will be serious pregnancy risks for both woman and fetus. Some clinical scenarios may be considered as relative or absolute contraindications to pregnancy (Table 2): these include moderate-to-severe lupus flare (including renal flare) or stroke within the past 6 months, pulmonary hypertension (estimated systolic pulmonary artery pressure $>50 \mathrm{mmHg}$, or symptomatic), moderateto-severe heart failure (left ventricular ejection fraction $<40 \%$ ), severe valvulopathy, severe restrictive lung disease (forced vital capacity $<50 \%$ predicted or $<1 \mathrm{~L}$ ), chronic kidney disease (CKD) stage 4-5 (creatinine $>2.5-2.8 \mathrm{mg} / \mathrm{dL}$, $>220-250 \mathrm{mmol} / \mathrm{L}$; estimated glomerular filtration rate [GFR] $<30 \mu \mathrm{L} / \mathrm{min}$ ), or uncontrolled hypertension. ${ }^{7,8,10,11}$ Women with previous severe early-onset preeclampsia ( $<28$ weeks) or HELLP syndrome (a form of severe preeclampsia: hemolysis, elevated liver enzymes, and low platelet count $)^{12,13}$ despite therapy with aspirin plus heparin must also be considered an extremely high-risk group. ${ }^{7,8,10,11}$

\section{Prepregnancy challenge: appropriate counseling}

Stratification of women into these three groups enables an overall approach to management, with adjustment(s) for individual situations: $: 8$

1. SLE in remission, or stable low disease activity: medication should be reviewed and adjusted as necessary; those women should be advised that it is safe to plan a pregnancy.

2. SLE at an early stage following recent diagnosis, or active disease: those women should be encouraged to postpone pregnancy and use effective contraception; medication should be reviewed with prescription of immunosuppressives (ideally hydroxychloroquine [HCQ] and/or azathioprine which will be safe in pregnancy); further review(s) should be done to monitor progress; once SLE condition improves (or ideally, enters remission), the women should be advised that it is safe to plan a pregnancy.

3. Severe impairment of organ function and/or preexisting severe organ damage (Table 2): those women should be advised about the serious risks to health and pregnancyrelated risks; pregnancy should be discouraged; alternatives, including adoption and surrogacy (own or donor eggs), should be discussed.

Once the women are stable with inactive disease, discussion of potential medical and obstetric risks, and appropriate planning for pregnancy, can be undertaken. Women should be reassured that while pregnancy with SLE is generally considered a high-risk pregnancy, a tailored management approach with close multidisciplinary surveillance in pregnancy and the puerperium will result in high rates of successful pregnancies. ${ }^{8}$

For a woman with SLE, the medical risks are the possible effect(s) of pregnancy upon her SLE (flares, progressive organ damage). The obstetric risks are due to the effects of SLE upon her pregnancy for her (preeclampsia, VTE) and the fetus (miscarriage, IUGR, preterm birth, stillbirth, NLS); any previous obstetric history will also suggest the likelihood of complications in future pregnancies.

Women with active disease (current, or during 6 months prior to conception), lupus nephritis, or cardiac/lung involvement are at higher risk of flares, and medical and/or obstetric complications during their pregnancy. Women with thrombotic and/or obstetric antiphospholipid syndrome (APS) are at an increased risk of VTE and/or pregnancy loss, and may require aspirin and/or low-molecular weight heparin (LMWH) during pregnancy. Women with anti-Ro and/or anti-La antibodies should be counseled about the risks of fetal-neonatal CHB and/or NLE (Table 3). ${ }^{9,14,15}$

It is important to distinguish between aPL and APS. The international consensus statement on APS classification includes laboratory and clinical criteria. A diagnosis of aPL

Table 3 Factors affecting pregnancy outcomes in SLE

Disease activity

Lupus nephritis (both in terms of hypertension and renal impairment)

Anti-Ro/anti-La antibodies

Antiphospholipid syndrome

Cardiac/lung involvement

Note: Data from ${ }^{9,14,15}$.

Abbreviation: SLE, systemic lupus erythematosus. 
requires two positive tests, $>12$ weeks apart, of at least one of lupus anticoagulant, anticardiolipin antibody (medium/high titer), and anti-beta2-glycoprotein I antibody. ${ }^{16}$ aPL may be present in up to $40 \%$ of patients with SLE, and $8 \%-10 \%$ of the normal population. ${ }^{17}$

APS is diagnosed if there are aPL with additional clinical features of thrombotic or obstetric complications. Thrombotic APS involves one or more clinical episodes of arterial, venous, or small-vessel thrombosis. Obstetric APS involves the following: one or more adverse pregnancy outcomes (unexplained fetal death(s) $\geq 10$ weeks of gestation; premature birth(s) of a morphologically normal fetus $<34$ weeks due to preeclampsia, eclampsia, or placental insufficiency (including birthweight less than tenth centile); or three or more unexplained consecutive spontaneous miscarriages $<10$ weeks). ${ }^{16}$

Soh et al compared pregnancy outcomes between women with aPL and obstetric APS. Both groups received aspirin in pregnancy; women with obstetric APS also received LMWH if they were at high risk of VTE, had previous late pregnancy complications, or previous adverse outcome(s) despite aspirin use. Women with aPL had similar obstetric outcomes to controls, whereas those with obstetric APS had a fourfold higher rate of pregnancy-induced hypertension or preeclampsia and a fivefold higher rate of pregnancy loss, with significantly lower birthweight and increased rate of SGA. They thus recommend that women who only have aPL should receive aspirin, but not routine LMWH solely for aPL, and can be reassured and managed as those with normal pregnancy. ${ }^{17}$

aPL should also be considered in its context as risk factor for thromboembolism. If there are three other risk factors, the woman may be considered for antenatal LMWH; if there are two other risk factors, LMWH may be considered from 28 weeks; if there is one other risk factor, postnatal LMWH may be considered for 10 days. Any woman receiving antenatal LMWH should continue this for 6 weeks postpartum. ${ }^{18}$

Women with thrombotic APS (who will often be on long-term oral anticoagulation) should be offered thromboprophylaxis with higher dose LMWH antenatally and for 6 weeks postpartum, or until returned to oral anticoagulant therapy after delivery. ${ }^{19}$ During pregnancy, these women should be managed in collaboration with a hematologist and/ or rheumatologist with expertise in this area. ${ }^{18}$

Women with obstetric APS with recurrent miscarriage $<10$ weeks should take aspirin from preconception. If they have previously miscarried while taking aspirin, LMWH should be added from the confirmation of pregnancy. Consideration may be given to discontinuing this either at 12 weeks or at 20 weeks if uterine artery waveform is normal at the anomaly scan. ${ }^{19}$

Women with obstetric APS with miscarriage $\geq 10$ weeks of gestation or with premature birth(s) of a morphologically normal fetus $<34$ weeks due to preeclampsia, eclampsia, or placental insufficiency (including birthweight less than tenth centile) should take aspirin from preconception with LMWH added from confirmation of pregnancy. This should be continued antenatally and for 6 weeks postpartum. ${ }^{19}$

Other risk factors have been identified for pregnancies in women with SLE; in many cases, these relate to the factors listed in Table 3, predominantly complications from lupus nephritis. They include clinical factors (hypertension, nonreversible organ damage particularly CKD) and biochemical factors (hypocomplementemia, proteinuria $>500 \mathrm{mg} / 24$ hours, thrombocytopenia, GFR $<60 \mathrm{~mL} / \mathrm{min} /$ $1.73 \mathrm{~m}^{2}$, raised creatinine particularly $>2.5-2.8 \mathrm{mg} / \mathrm{dL}$ and $\geq 220-250 \mu \mathrm{mol} / \mathrm{L})^{8,10,20-24}$

In addition to changing or stopping medication that could be harmful in pregnancy, and starting additional medication such as antihypertensives, all women with SLE should be advised to take low-dose aspirin $(75 \mathrm{mg})$ from 12 weeks and throughout pregnancy to reduce their risk of developing preeclampsia. ${ }^{12,25}$

Appropriate vitamins and minerals should also be prescribed. Any woman planning to conceive should take $400 \mu \mathrm{g}$ folic acid daily to reduce the risk of fetal neural tube defects, ideally for 3 months preconception and the first 3 months of pregnancy. ${ }^{25}$ If she has had methotrexate in the previous 3 months, ${ }^{10}$ and diabetes, obesity, or any history (or family history) of of neural tube defects, then she should be prescribed high-dose $\left(5 \mathrm{mg}\right.$ ) folic acid. ${ }^{27}$ All women should also take $10 \mu \mathrm{g}$ vitamin D daily for bone and teeth health and development. Women at increased risk of vitamin D deficiency should have levels checked as they may require a higher dose. ${ }^{26}$ Calcium therapy is also recommended. Previously, this was for women on long-term corticosteroids or LMWH, or at high risk of osteoporosis. However, a recent meta-analysis of 13 randomized trials involving $>15,000$ women showed that at least $1 \mathrm{~g}$ of calcium daily in pregnancy (vs placebo) resulted in $>50 \%$ reduction in the risk of preeclampsia, and $25 \%$ reduction in the risk of preterm delivery. As women with SLE are at an increased risk of preeclampsia, we recommend calcium supplementation for all. ${ }^{28}$

Finally, general preconception advise should be given. Smoking cessation should be recommended, with referral to a smoking cessation program. Alcohol intake should be reduced or stopped; recreational drugs should also be 
stopped. These measures will improve her health, her chance of conceiving, and the health of her fetus. If a woman is overweight, she should be counseled about the importance of weight loss, ideally to normal body mass index (BMI) range $\left(17.5-24.9 \mathrm{~kg} / \mathrm{m}^{2}\right)$ as this will also improve her chance of conceiving, and reduce her risk of miscarriage and other pregnancy complications (including gestational diabetes and VTE). Rubella immunity should be checked, and vaccination with measles, mumps, and rubella vaccine should be offered to nonimmune women (ensuring that they are not currently pregnant, and advising avoidance of pregnancy for a month). Hemoglobinopathy assessment may be undertaken in women at risk of being carriers of sickle cell disease or thalassemia..$^{8,10}$

Fertility is generally not affected in patients with SLE, other than those with amenorrhea secondary to severe flares, CKD 3-5 (estimated GFR $<60 \mathrm{~mL} / \mathrm{min}$ ), or ovarian failure following cyclophosphamide. ${ }^{11}$ Women with SLE who have not conceived spontaneously and wish to undergo assisted reproduction techniques, including in vitro fertilization, should be counseled about the increased risk of disease flare and VTE (especially women with ovarian hyperstimulation syndrome and/or other prothrombotic risk factors). Identification of high-risk patients (Table 3 ), precycle counseling, and adequate thromboprophylaxis and surveillance are mandatory. In patients with APS, safer approaches may include ovarian stimulation with clomiphene, single embryo transfer, ongoing therapy (appropriate for pregnancy) to avoid disease flare, avoidance of ovarian hyperstimulation syndrome, and use of natural estradiol and/or progestogens administered vaginally. $^{11}$

For women who are advised to delay pregnancy, appropriate and reliable methods of contraception should be discussed. One study of 212 women at a lupus center showed that $46 \%$ had faced some risks of unintended pregnancy, with $23 \%$ having unprotected sex "most of the time". ${ }^{29}$

\section{Prepregnancy challenge: appropriate medication}

The use of medication in pregnancy is often treated with understandable caution by manufacturers and medication administrative bodies, and is usually based on data from retrospective case series, isolated case reports, and animal studies.

The European League Against Rheumatism task force recently reviewed the use of antirheumatic drugs before and during pregnancy, and during lactation, including registry data and a systematic review of the literature. In 2016, they produced four overarching principles, as well as statements ("points to consider"), on the use of these drugs during pregnancy and lactation. ${ }^{30}$ The EULAR overarching principles include the importance of family planning, the aim to prevent or suppress disease activity in the mother whilst minimizing harm to the fetus/child, the balancing of risks of medication against risks of untreated disease, and the importance of multidisciplinary input (rheumatologist, obstetrician, patient) when making decisions about treatment. ${ }^{30}$ The British Society for Rheumatology and British Health Professionals in Rheumatology also produced evidence-based guidelines in 2016 on prescribing drugs in pregnancy and breastfeeding. ${ }^{31,32}$

Details of medications and safety information are provided in Table 4: anti-inflammatories and analgesics, corticosteroids (non-fluorinated), and immunosuppressants (including anti-malarials)..$^{8,9,31-35}$

Medications which are safe throughout pregnancy (all trimesters) and during lactation to maintain remission and/ or treat flare are HCQ, azathioprine, cyclosporine, and tacrolimus. ${ }^{9,30}$ The aim should be to use steroid-sparing immunosuppressants (such as azathioprine) with ideally no steroids or else the lowest effective maintenance dose (ideally $<7.5 \mathrm{mg}$ prednisolone per day). ${ }^{9,30}$ Corticosteroids are safe to control active disease symptoms; aspirin and paracetamol are also safe. ${ }^{9,30}$ Methotrexate, mycophenolate mofetil, and cyclophosphamide must be discontinued before pregnancy due to proven teratogenicity, and should not be used during lactation. ${ }^{30}$

In general, mild flares during pregnancy can be treated with HCQ and/or low-dose oral steroids (also possibly with nonsteroidal anti-inflammatory drugs if first or second trimester and previously responsive). Moderate or severe disease may require the use of pulsed intravenous methylprednisolone or high-dose oral steroids, followed by rapid reduction to low-dose maintenance oral steroids in combination with safe immunosuppressants. Intravenous immunoglobulin (IVIg) may also be necessary.

More severe cases may require a risk/benefit assessment and prioritization of maternal welfare over fetal concerns, with use of stronger agents such as mycophenolate mofetil or cyclophosphamide, and consideration of preterm delivery. Management of lupus nephritis flares during pregnancy may include pulsed steroid followed by a combination of prednisolone, HCQ, azathioprine, or tacrolimus.

Antihypertensive medication for preexisting or pregnancy-induced hypertension/preeclampsia may include labetalol, methyldopa, and/or nifedipine as safe first-line treatment (singly or in combination) at any stage of conception, pregnancy, or lactation. ${ }^{12,19}$ Second-line treatments during 


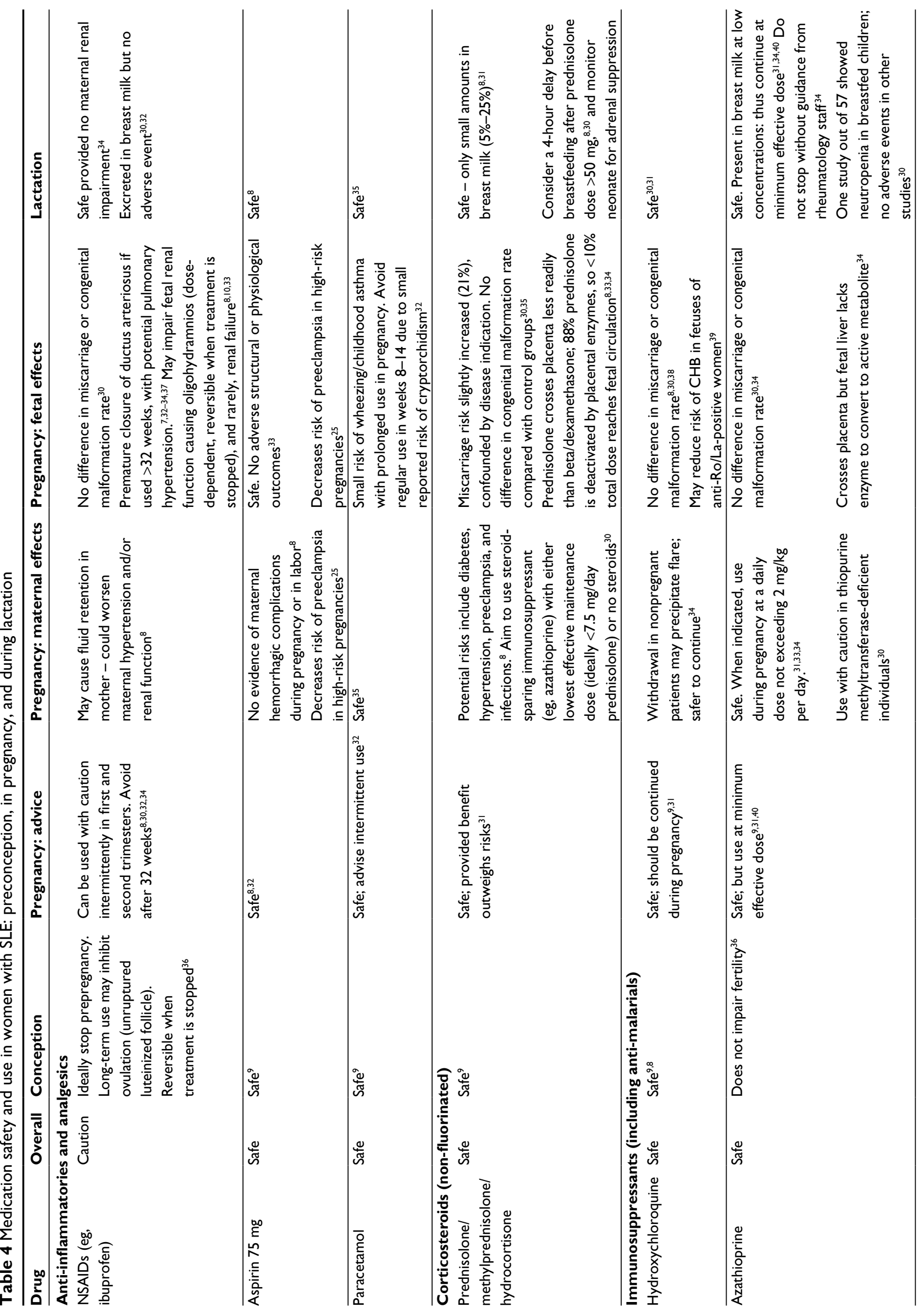




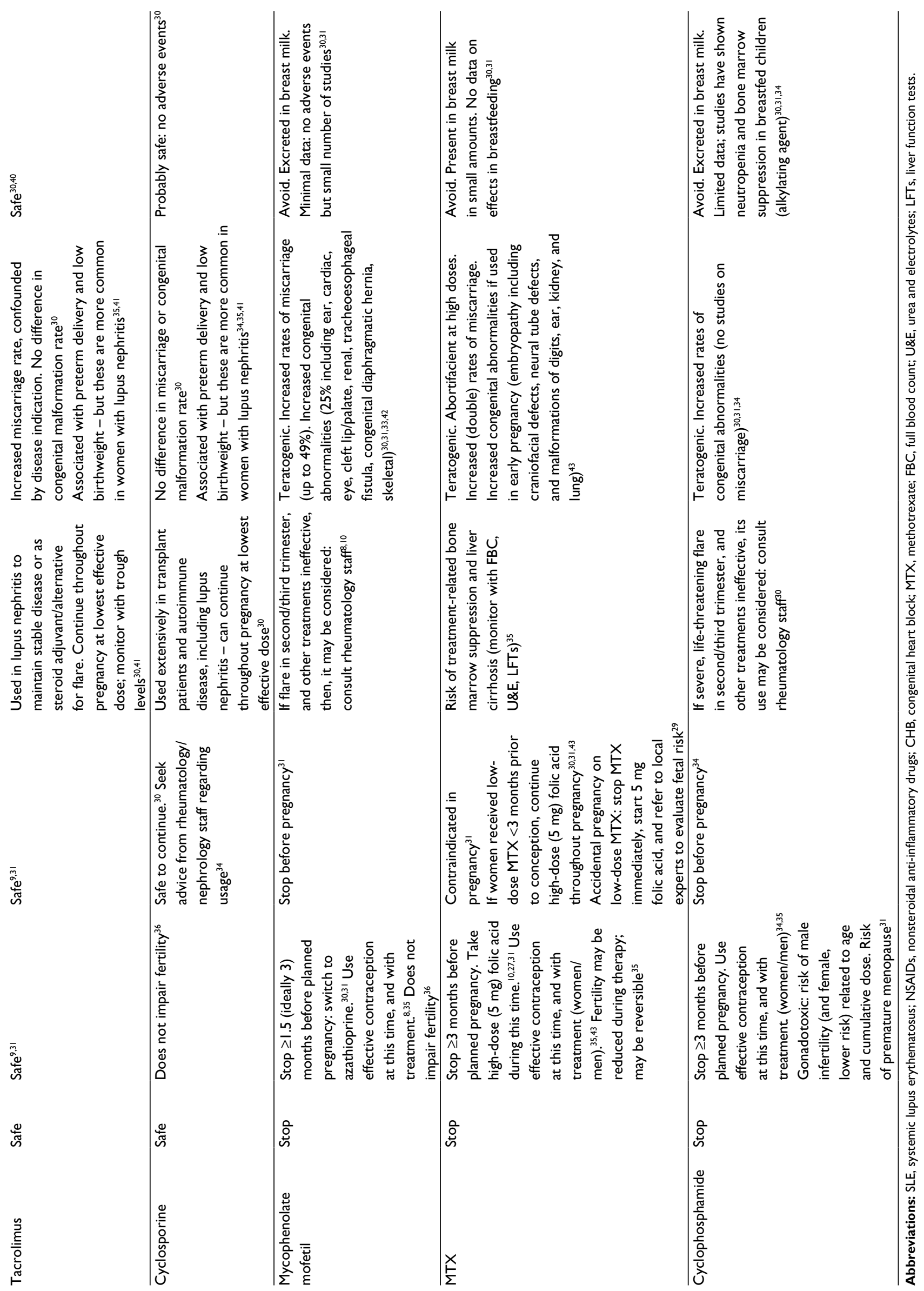


pregnancy include hydralazine or doxazosin. ${ }^{19}$ Angiotensinconverting enzyme (ACE) inhibitors and/or angiotensin II receptor blockers should be stopped preconception as they are associated with an increased risk of congenital abnormalities. ${ }^{12}$ Some women with renal disease who are planning to conceive may, after specialist input, continue ACE inhibitors until very early in pregnancy ${ }^{8}$ (eg, first positive pregnancy test after a missed menstrual period) and then switch to another (first-line) antihypertensive, ${ }^{32}$ as months without the renal protective effects of ACE inhibitors may be deleterious to their long-term health. ACE inhibitors (eg, enalapril) can be used safely postpartum, including during lactation. ${ }^{19}$

\section{Antenatal challenge: maternal lupus flares}

Pregnancy is considered a high-risk time in SLE patients, although studies provide conflicting results as to whether flares are more common or of unchanged frequency. ${ }^{44}$ The changes in hormonal profiles in pregnancy induce a shift from Th1 to Th2 lymphocyte dominance; thus, Th2-response autoimmune disorders, such as SLE, are more likely to flare. ${ }^{10}$

The LUMINA Study Group in the US looked at outcomes in SLE patients including assessment of cumulative and irreversible damage, irrespective of cause, in 12 organ systems. They found significant postpartum damage accrual. This was due to disease activity levels and the degree of damage prior to pregnancy, total disease duration, and pregnancy duration, rather than due to pregnancy per se. The strongest predictor of postpartum damage accrual was having more active disease prior to pregnancy. ${ }^{45}$

Overall, the risk of flare (antenatal or postpartum) appears to be dependent on disease activity 6-12 months prior to conception. Women with quiescent SLE over this period have less risk of flare during pregnancy, whereas women with active SLE have a high risk of flare..$^{10}$ Most flares are nonsevere ${ }^{7}$ with articular, dermatological, and/or mild hematological involvement. These are usually well controlled with shortterm introduction or increase of oral steroids. Nonetheless, severe flares with major organ involvement may occur; ${ }^{10}$ the Hopkins Lupus Center in the US has suggested that renal, as well as hematological, flares may be more common in pregnancy. ${ }^{44}$

Lupus nephritis at conception confers a higher risk of flare during pregnancy, even in women in remission. One study of 113 pregnancies in 81 women with preexisting lupus nephritis showed a $30 \%$ flare rate during pregnancy or postpartum; ${ }^{23}$ others suggest up to $60 \%$ likelihood. ${ }^{24}$ In women with previous lupus nephritis, pregnancy does not seem to endanger long-term renal function, although the higher the baseline creatinine, the greater the risk of deterioration. ${ }^{23}$ Women with creatinine $>2.5-2.8 \mathrm{mg} / \mathrm{dL}(>220-250 \mu \mathrm{mol} / \mathrm{L})$ are most likely to suffer postpartum renal function decline. ${ }^{24}$

In addition to renal complications, women may also suffer from a worsening of hypertension, or onset of new hypertension and/or preeclampsia. The risk of VTE is also increased.

A recent systematic review established the protective effect of HCQ in terms of organ damage, flares, VTE, bone mass loss, and long-term survival in the general SLE population, as well as the potential to prevent disease activity in pregnant women. ${ }^{46}$ Two prospective studies corroborated these findings, suggesting that women who had taken HCQ throughout pregnancy had lower disease activity scores and lower prednisolone doses at the end of pregnancy, whereas those who discontinued HCQ, or did not take it at all, had higher activity scores, more flares, and required higher doses of steroids. ${ }^{38,47}$ HCQ may also offer fetal benefits in women who have anti-Ro/La antibodies. ${ }^{39}$

Distinguishing pregnancy-associated signs and symptoms from those of SLE may be difficult. Involvement of, and assessment by, experienced physicians is important. Fatigue, mild arthralgia, hair loss, dyspnea, headaches, malar and palmar erythema, edema, anemia, and thrombocytopenia represent common ambiguous manifestations. ${ }^{7}$

Baseline bloods (FBC, U\&E, creatinine, LFTs, serological profile) and urinalysis with proteinuria quantification should ideally be obtained prepregnancy and again in early pregnancy. These can help to monitor disease status and identify flares. In pregnancy, erythrocyte sedimentation rate is usually raised due to higher fibrinogen production in the liver; ${ }^{19,48}$ hence, it is not considered a valid marker of disease activity in pregnancy. Serum C3 and C4 levels also rise in pregnancy due to increased liver production, ${ }^{7}$ so they may remain within normal range even in women with active SLE. Relative variation, rather than absolute levels, of C3 and C4 should be assessed. A fall of $\geq 25 \%$ in serum complement levels during pregnancy may suggest lupus flare. ${ }^{49}$

In patients with permanent significant protein loss due to previous lupus nephritis, proteinuria may increase during pregnancy due to increased renal blood flow, without indicating active nephritis. ${ }^{7}$ This may be more pronounced in patients who stop ACE inhibitors and/or angiotensin II receptor blockers shortly before or early in pregnancy. Up to a doubling of proteinuria from the baseline level in early pregnancy is to be expected. ${ }^{10}$ Thromboprophylaxis with LMWH may be required if there is significant proteinuria; expert opinion suggests a threshold of protein:creatinine ratio 
Table 5 Differentiating infection from inflammation (flare) in SLE patients

\begin{tabular}{lll}
\hline $\begin{array}{l}\text { Features } \\
\text { suggestive of } \\
\text { infection }\end{array}$ & $\begin{array}{l}\text { Features suggestive of } \\
\text { inflammation (flare) }\end{array}$ & $\begin{array}{l}\text { Features which } \\
\text { may be common } \\
\text { to both }\end{array}$ \\
\hline Raised CRP & $\begin{array}{l}\text { Normal CRP (may be } \\
\text { raised in pericarditis, } \\
\text { pneumonitis, or arthritis) }\end{array}$ & $\begin{array}{l}\text { Symptoms (nausea, } \\
\text { vomiting, pleuritic } \\
\text { pain, arthralgia) }\end{array}$ \\
Response to & Fever \\
Reised WCC & $\begin{array}{l}\text { immunosuppression } \\
\text { Response to }\end{array}$ & $\begin{array}{l}\text { Low complement } \\
\text { (C3 and C4) }\end{array}$ \\
$\begin{array}{l}\text { Raised } \\
\text { procalcitonin } \\
\text { (intensive care }\end{array}$ & Raised dsDNA antibodies & \\
setting) & & \\
\hline
\end{tabular}

Note: Data from Ateka-Burrutia et al, ${ }^{8}$ and Beca et al. ${ }^{52}$

Abbreviations: SLE, systemic lupus erythematosus; CRP, C-reactive protein; WCC, white cell count.

(PCR) $>100-200 \mathrm{mg} / \mathrm{mmol}$ or 24-hour collection $>1-2 \mathrm{~g} /$ day during pregnancy. ${ }^{50,51}$

It can also be difficult to distinguish between infection and inflammation (flare) in patients with SLE (Table 5), ${ }^{8,52}$ This is important as sepsis is a prominent cause of indirect maternal mortality, as well as mortality in nonpregnant SLE patients. If there is any diagnostic doubt, it may be worth using IVIg for first-line treatment as this will dampen inflammation but not worsen infection (unlike high-dose steroids). ${ }^{8}$

\section{Antenatal challenge: maternal obstetric complications}

A 2008 study from the US involving $>13,000$ deliveries in women with a diagnosis of SLE showed that SLE patients have a 17 - to 20 -fold increased risk of maternal mortality (325/100,000 live births, or 0.32\% risk in all SLE pregnancies). However, it should be noted that while being higher than the background rate in uncomplicated pregnant women, this is actually several-fold lower than the annual mortality rate in nonpregnant SLE patients $(0.8 \%-3.2 \%){ }^{20}$

Medical complications such as stroke, pulmonary embolism (PE), deep vein thrombosis (DVT), major infections, bleeding, and thrombocytopenia are two to eight times more frequent among women with SLE. ${ }^{20}$

After controlling for confounding factors such as maternal age, women with SLE still had higher risks of pulmonary hypertension, renal failure, and thrombophilia (thrombophilia predominantly due to APS) (odds ratio [OR] 2.6, 3.7, and 20.9, respectively). The risk of major infections (sepsis, pneumonia) was also increased (OR 3.5-4.3). Hematologic complications including anemia, thrombocytopenia, and need for blood transfusion were increased (OR 12.6, 8.3, and 3.6, respectively), but the risks of antepartum and postpartum hemorrhage were less elevated (OR 1.8 and 1.2). The risk of thrombotic events (DVT, PE, and stroke: OR 7.9, 5.5, and 6.5 , respectively) was more than tenfold higher in women with SLE. ${ }^{20}$

It is important to note that while pregnancy can pose risks to a woman with SLE (which are certainly higher when compared with a low-risk pregnant population), the risks may not be higher than in any other year of that woman's life, given her underlying diagnosis of SLE. ${ }^{20}$

Conversely, those with SLE in remission without major organ involvement, or those with cutaneous lupus erythematosus only, are likely to have a normal pregnancy with pregnancy outcomes comparable to those of the healthy population. ${ }^{7,10}$

The risk of preeclampsia is noticeably increased in women with SLE. Twenty-three percent or more may develop preeclampsia: two- to fourfold higher than the general population. ${ }^{8,20,25}$ This risk may be even greater if they have had preeclampsia before (OR 7.19) or have APS (OR 9.72), ${ }^{.3}$ patients with lupus nephritis, chronic hypertension, and renal impairment and women on high-dose oral steroids are also at particular risk (the latter is likely to be reflective of disease activity, although steroids do increase blood pressure) after adjustment for maternal age. ${ }^{20,21,54}$ Other independent risk factors include hypertensive disease during a previous pregnancy, CKD, diabetes (type 1 or type 2), or two or more factors of first pregnancy, age $\geq 40$ years, pregnancy interval $\geq 10$ years, BMI $>35 \mathrm{~kg} / \mathrm{m}^{2}$ at first visit, a family history of preeclampsia, or multiple pregnancy. ${ }^{12}$ Women with SLE also have a fourfold increased risk of developing eclampsia compared with the general population, although absolute numbers remain small $(0.5 \%$ vs $0.09 \%) .{ }^{20}$

A meta-analysis showed a $10 \%$ risk reduction in preeclampsia, preterm delivery $<34$ weeks, perinatal death, birthweight less than tenth centile, or serious pregnancy outcomes (maternal death, development of preeclampsia; preterm delivery, SGA fetus, stillbirth, or neonatal death) if women take aspirin from $<16$ weeks of gestation throughout pregnancy. This is thus recommended for all women with SLE in pregnancy. ${ }^{12,25}$

One particular challenge in pregnant patients with SLE is differentiating preeclampsia from lupus nephritis (Table 6). ${ }^{7,82,45}$ This may be challenging as they have symptoms in common and may also coexist: women with preexisting renal disease are at higher risk of hypertensive complications during pregnancy, including preeclampsia.,24 
Table 6 Differentiating lupus flare from preeclampsia

\begin{tabular}{lll}
\hline Features suggestive of preeclampsia & Features suggestive of lupus flare & $\begin{array}{l}\text { Features that may be common } \\
\text { to both }\end{array}$ \\
\hline Severe headache & Onset $<20$ weeks (more suggestive of SLE) & Hypertension \\
Visual symptoms (including flashing lights) & Active urinary sediment/cellular casts & Worsening proteinuria \\
Epigastric or right upper quadrant tenderness & Hematuria & Edema \\
Clonus (>2 beats) & Low/falling complement levels & Renal impairment \\
Abnormal LFTs & High/increasing anti-dsDNA antibodies & Thrombocytopenia \\
Rising uric acid level & Evidence of flare involving other organs & \\
Signs of hemolysis & & \\
Falling angiogenic factors (eg, PIGF, VEGF) & & \\
\hline Note: Data from $7,8,245.5$ & & \\
Abbreviations: SLE, Systemic lupus erythematosus; LFTs, liver function tests; PIGF, placental growth factor; VEGF, vascular endothelial growth factor.
\end{tabular}

A renal biopsy may be indicated if the result would affect management, usually by confirming or refuting a diagnosis (and/or class) of lupus nephritis. Special care should be taken due to higher bleeding risks after biopsy in pregnancy. ${ }^{8}$

New biomarkers, including angiogenic factors such as placental growth factor, may help to diagnose preeclampsia ${ }^{13}$ and may be particularly important in the challenging situation of identifying superimposed preeclampsia in patients, including those with SLE and those who have underlying hypertension and/or renal disease. A recent study showed that, from 20 to 42 weeks of gestation, lower maternal placental growth factor concentrations had high diagnostic accuracy for superimposed preeclampsia requiring delivery within 14 days. ${ }^{56}$

\section{Antenatal challenge: fetal complications}

The LUMINA Study Group in the US assessed the impact of SLE on pregnancy outcomes $(n=102)$. Thirty-six percent of women with SLE had a good pregnancy outcome (37/102), and $64 \%$ had an adverse outcome (65/102). As a fraction of the whole group, approximately a fifth had miscarriage $<20$ weeks $(n=21)$, termination of pregnancy $(n=20)$, or preterm birth $<34$ weeks $(\mathrm{n}=19)$, with a tenth suffering stillbirth $(\mathrm{n}=5)$. It is, however, important to note that the reason for the termination (whether maternal request, or therapeutic for maternal and/or fetal indications) was not recorded. The authors also suggest that many patients in their study population may have lacked access to specialized obstetric-rheumatology care, which is known to have beneficial effects on pregnancy outcomes. ${ }^{21}$

A 2008 literature review suggested a comparable mean fetal death rate (miscarriage plus stillbirth) of $19.5 \%$ in women with SLE, although with a very wide range $(4 \%-43 \%) .{ }^{57}$ The rate was higher in patients with active SLE at conception $(25 \%-52 \%) ;{ }^{57}$ similarly high rates $(53 \%)$ were seen in another cohort of lupus nephritis patients. ${ }^{58}$ Patients with inactive SLE at conception had much lower rates of fetal loss $(8 \%-12 \%)$, comparable with healthy women. ${ }^{57}$ One paper reported a fourfold increase in the stillbirth rate in SLE patients, although this halved after excluding patients with CNS lupus. ${ }^{59} \mathrm{~A}$ recent American study of 83 pregnancies in women with SLE showed a pregnancy loss rate of $17 \%$, comparable with the US average of $16.1 \%{ }^{60}$

Ongoing pregnancies in patients with SLE carry increased risks of SGA and/or IUGR (6\%-35\%) ${ }^{8,20,21}$ Monitoring fetal growth with ultrasound scans is thus important in any women with SLE with risk factors for poor pregnancy outcome (Table 3).

The rate of preterm labor and delivery ( $<37$ weeks) is also increased to $21 \%-36 \%$ in pregnancies in women with SLE (higher in women with more active or complicated disease), compared with $12 \%$ national average..$^{20,21,58,60}$ However, the figures for spontaneous labor, induced labor, and prelabor cesarean section within this preterm group were not available. In one study of pregnancies in SLE patients, mean pregnancy duration was 29.6 weeks. ${ }^{45}$ Renal involvement conveys a significant increase in the rate of lower mean birthweight babies and in preterm delivery compared with SLE patients without renal involvement. ${ }^{54,61}$

\section{Antenatal/postnatal challenge: neonatal lupus}

NLS is a rare complication affecting children born to mothers with anti-Ro/La antibodies, found in $25 \%-40 \%$ (antiRo) and $10 \%-15 \%$ (anti-La) women with SLE $^{62}$ as well as other autoimmune diseases (eg, Sjögren's syndrome). These IgG antibodies are actively transported across the placenta from 16 to 30 weeks of gestation. ${ }^{7}$ There are two main forms of NLS: NLE and CHB. Rarer forms include hepatic and hematologic. Forms may coexist: for example, a child with CHB may also have skin manifestations. Cutaneous, hepatic, and hematologic symptoms are transient; CHB is permanent. ${ }^{63}$ 
NLE occurs in $5 \%$ of children born to women with anti$\mathrm{Ro} / \mathrm{La}$ antibodies. It generally presents within the first 2 weeks of life as erythematous geographical lesions in light-exposed areas, resembling those seen in subacute cutaneous lupus. The rash usually disappears within 6 months as the maternal antibodies are cleared, and does not leave residual scarring. ${ }^{63,64}$

The US Research Registry for Neonatal Lupus has assessed neonatal outcomes in 58 women with 77 pregnancies following a child with NLE. Forty-nine percent of these pregnancies were affected with NLS, and 51\% were unaffected. Of the total 77 pregnancies, $30 \%(n=23)$ had NLE, $18 \%$ (14) had CHB \pm NLE, and $1 \%$ (one) had hematologic/ hepatic neonatal lupus. Limiting assessment to the first child born during prospective follow-up after a child with NLE (to remove possible bias due to multiple pregnancies in the same families) showed $36 \%$ of pregnancies to be affected in total: $23 \%$ with NLE and $13 \%$ with CHB \pm NLE. Sixtyfour percent of pregnancies were unaffected. There were no significant differences in maternal risk factors (age, race/ ethnicity, anti-La status, use of steroids, breastfeeding) or in fetal sex for having a subsequent child with NLS. ${ }^{64}$

CHB is a more serious form of NLS, affecting $2 \%$ of newborns of anti-Ro-positive women with known connective tissue diseases ${ }^{65,66}$ - it remains a very rare condition in the general population, affecting 1:20,000 pregnancies. ${ }^{67}$ If an anti-Ro/La-positive woman has had a baby with CHB, then the risk to subsequent pregnancies increases to $18 \%$. The actual incidence may be higher as incomplete forms of CHB have been described, including first-degree heart block that can progress during childhood. Most children with CHB will need a permanent pacemaker $\left(47 \%-64 \%\right.$ in first year ${ }^{67,68}$ and $75 \%$ by 10 years $),{ }^{68}$ and up to $20 \%$ may die in the perinatal period. ${ }^{66,68}$ Neonatal CHB may also be the first presentation of otherwise asymptomatic connective tissue disease in up to $50 \%$ of women: all mothers of infants with $\mathrm{CHB}$ are tested for anti-Ro/La antibodies. ${ }^{69}$

A national review of antenatally diagnosed CHB in 87 pregnancies over 27 years found no cases detected at $<18$ weeks of gestation. Eighty-two percent were detected $<30$ weeks, with the majority at 20-24 weeks. Subanalysis of more recent data (1988-1997) showed that $90 \%$ were detected $<30$ weeks. In many cases, this detection followed an initially normal fetal heart rate. ${ }^{70} \mathrm{~A}$ French registry of $254 \mathrm{CHB}$ cases (1976-2014) showed that $94.4 \%$ were detected antenatally, with a median detection of 23 weeks (range 16-39 weeks). ${ }^{68}$

Ultrasound assessment of the fetal heart by a fetal medicine or fetal cardiology specialist should thus be performed at 18-20 weeks of pregnancy in all women with anti-Ro/La antibodies. This enables assessment of atrial and ventricular rates, cardiac anatomy and function, and the presence or absence of hydrops ${ }^{69}$ Many units rescan at 26-28 weeks and/or at interim visits. ${ }^{22}$

The fetal echo provides parental reassurance, but in terms of detection of heart block, it is more important for the fetal heart rate to be auscultated and recorded every 1-2 weeks at an antenatal clinic visit, with urgent referral to a fetal medicine unit or fetal cardiology service if a low fetal heart rate $(<110 \mathrm{bpm})$ is detected. There is an increased risk of hydrops and death if the rate is $<55 \mathrm{bpm} .^{71}$

If evidence of incomplete heart block, myocarditis, ascites, or hydrops is identified, then therapy with fetal steroids (dexamethasone or betamethasone) could be considered..$^{22,68}$ Three large studies found no benefit on feto-neonatal mortality ${ }^{68}$ one study of historical cases suggested an improvement in 1-year survival with steroid treatment. ${ }^{72}$ Given the potential maternal complications with steroid usage, this treatment is not routinely recommended. ${ }^{68}$ Irrespective, the development of hydrops in a fetus with CHB is an extremely poor prognostic sign, with a mortality rate of $83 \%-100 \%{ }^{68,72}$ The presence of endocardial fibroelastosis and/or a fetal heart rate of $<55 \mathrm{bpm}$ are also poor prognostic factors. ${ }^{69}$

It was previously thought that IVIg might help to reduce the rate of recurrent $\mathrm{CHB}$, but two clinical trials have failed to show this. ${ }^{73,74}$ However, a recent case-control study suggested a protective effect of HCQ on the development of cardiac manifestations in children born to anti-Ro/La-positive mothers. Retrospective data from three large international registers were assessed to identify women with anti-Ro/La antibodies who had a further pregnancy after a child with CHB. The recurrence rate was $7.5 \%$ in the group who took HCQ compared with $21.2 \%$ in the group who did not. ${ }^{39}$ Given the good safety profile and minimal side effects of HCQ in pregnancy, we recommend starting any women with anti-Ro/La-positive antibodies on HCQ (400 mg daily) either prepregnancy or as soon as possible $<16$ weeks of gestation, and continuing throughout pregnancy. ${ }^{22}$ They should still receive antenatal fetal assessment as outlined earlier.

\section{Antenatal challenge: management of pregnancy}

All women with SLE who are pregnant should be seen by, and have pregnancy care coordinated by, an obstetrician. They should also see a rheumatologist familiar with SLE in pregnancy at least once. Women should have routine pregnancy booking. In addition to booking blood tests (which include a FBC), baseline tests of renal and hepatic function, serological 
status (if unknown), and disease activity (C-reactive protein, anti-ds DNA, antibodies, complement) should be obtained. Women with previous lupus nephritis, other risk factors for poor pregnancy outcome (Table 3 ), or proteinuria on dipstick testing should also have baseline urinary protein quantified by PCR or 24-hour collection. ${ }^{8}$

Women should be seen in the first trimester by both obstetrician and rheumatologist to ascertain disease status and pregnancy risk. The frequency of subsequent antenatal and/or rheumatology clinic visits will depend on these risks, plus any factors in the obstetric history and the development of any complications in the current pregnancy. As a guide, women should be reviewed every 4 weeks from 16 to 28 weeks, every 2 weeks from 28 to 34 weeks, and every week from 34 weeks. ${ }^{8}$ Each visit should document the presence or absence of flare or preeclampsia symptoms, plus blood pressure, dipstick urinalysis, symphysial-fundal height, and fetal heart rate.

All women with SLE should take low-dose aspirin ( $75 \mathrm{mg}$ ) from 12 weeks of pregnancy to reduce their risk of preeclampsia. ${ }^{12,25}$ VTE risk should also be assessed at their first visit and throughout pregnancy; those at high risk should receive thromboprophylaxis with $\mathrm{LMWH}$ and be educated about DVT/PE symptoms. ${ }^{18}$ Antenatal obstetric anesthetic review should be arranged for women taking therapeutic or high-dose (twice daily) LMWH during pregnancy to discuss analgesia options and document an anesthetic plan for labor and delivery. Any patients with reduced respiratory function or cardiorespiratory compromise should also have an antenatal obstetric anesthetic review.

Women who have mild, stable disease with no other SLE risk factors for a more complicated pregnancy (Table 3) may be suitable for ongoing management by obstetricians and midwives, with further medical/rheumatological input if complications ensue. ${ }^{8}$

Women with active disease, or those at high risk of complications, should have regular input from both obstetrician and rheumatologist, plus other specialists (eg, nephrologist, cardiologist) depending on disease manifestations. Ideally, they should be managed in a multidisciplinary obstetric-rheumatology clinic throughout pregnancy to minimize appointments and provide continuity of, and comprehensive, care.

Women with previous hypertension, preeclampsia, and/or renal disease should have more frequent blood pressure and urinalysis checks than described earlier. ${ }^{8}$ These additional reviews could be with a general practitioner/family physician, or midwife, with referral if any concerns. Proteinuria on dipstick analysis would necessitate confirmation and quantification by either PCR or 24-hour collection.
Women on steroids, or those with previous gestational diabetes, should have screening for gestational diabetes, ${ }^{8}$ ideally at 16 weeks, and if negative, again at 26-28 weeks. Depending on local policy, this may be by random blood glucose, glycosylated hemoglobin (HbA1c), oral glucose tolerance test, or fingerprick blood sugar monitoring.

Women with severe or active SLE should have blood tests (as per baseline, described earlier) repeated every 4-8 weeks during pregnancy, with interim testing if there are any newonset symptoms suggestive of flare or preeclampsia. ${ }^{8}$

Women should be offered routine pregnancy screening and scans, usually a first trimester scan and trisomy screen (11-13 weeks) and a fetal anomaly scan (18-22 weeks). Uterine artery Doppler measurements at the latter may be helpful to stratify risk: a normal result has a high negative predictive value for preeclampsia and IUGR, while an abnormal result suggests a higher risk of these conditions. ${ }^{75}$

Ultrasound scans to assess fetal growth and well-being (amniotic fluid assessment, Doppler measurements of fetal blood flow) should be performed in patients with active disease or previous complex obstetric histories: a standard approach might be a late second trimester (26-28 weeks) and mid-third trimester (34-36 weeks) scan. If there are particular concerns, then scans may be performed every 2-4 weeks, with weekly (or more frequent) assessment of amniotic fluid and Dopplers, and liaison with a fetal medicine consultant if there are signs of fetal compromise. Such scans may also be requested for clinical concerns about fetal growth or wellbeing (reduced symphysial-fundal height, or reduced fetal movements) or in the event of a complication including lupus flare or preeclampsia.

Women with anti-Ro/La antibodies should have additional monitoring due to the risk of fetal CHB. ${ }^{76}$ They should be referred for fetal echocardiography at 18-20 weeks and again at 26-28 weeks. The fetal heart should be auscultated every 1-2 weeks, with the rate documented. Any fetal heart rate abnormality, particularly a slow heart rate, should trigger an urgent referral to a tertiary center. Routine antenatal clinic appointment frequency can resume after 28 weeks as the chance of developing $\mathrm{CHB}$ is markedly reduced in the third trimester; ${ }^{68}$ the fetal heart rate should still be documented.

Finally, it should be remembered that $30 \%-50 \%$ of pregnancies are unplanned. Women with SLE, therefore, may conceive during teratogenic drug therapy. Some patients may choose termination of pregnancy; others will wish to continue. For the latter, ultrasound confirmation of gestational age, and determination of exact exposure dates, is extremely important to enable individual risk assessment and accurate counseling. Detailed ultrasound scans by fetal medicine 
experts to screen for major structural abnormalities can be undertaken at 12 and 20 weeks of gestation (as per standard UK screening), with the option of an "early" anomaly scan at 16 weeks. Invasive testing (chorionic villous sampling or amniocentesis) is not indicated solely for teratogenic drug exposure but would be offered to patients with high-risk trisomy screening, or with fetal structural abnormalities. ${ }^{30}$

\section{Intrapartum challenge: managing labor}

Women with SLE have an increased risk of preterm delivery. This may be spontaneous or may be iatrogenic due to maternal and/or fetal concerns (severe lupus flare, preeclampsia, IUGR). A woman in preterm labor with a fetus in a cephalic or breech presentation should anticipate a spontaneous vaginal delivery, subject to any intrapartum complications; a women being delivered preterm for maternal and/or fetal concerns may require a cesarean section.

If the fetus is in $24^{+0}-34^{+6}$ weeks of gestation, two maternal intramuscular steroid injections (betamethasone or dexamethasone) should be given to aid fetal lung maturation, ideally $>24$ hours but $<7$ days before delivery. ${ }^{77}$ This is independent of any maternal steroids as prednisolone only reaches the fetus in minimal quantities $(<10 \%$ maternal dose) due to metabolism by the placenta. ${ }^{33}$ If the gestational age is $<32$ weeks, there is evidence that magnesium sulfate may have fetal neuroprotective benefits (it may also be given to women with severe preeclampsia to prevent eclampsia). ${ }^{78}$

A woman with SLE in spontaneous labor at term with a fetus in a cephalic presentation should aim for a spontaneous vaginal delivery. Studies have shown that women with SLE are more likely to be delivered by cesarean section ( $>33 \%$, OR 1.7): this may be for many reasons, and at a variety of gestations. ${ }^{20,21}$ Cesarean section is an additional risk factor for VTE (OR 2-6.7 compared with vaginal birth), ${ }^{18}$ carries risks of bleeding and infection, and has implications for future pregnancies. We would thus recommend that Cesarean sections should only be performed for obstetric indications (maternal and/or fetal concerns).

It may be necessary to adjust maternal medication for labor. If a woman is taking long-term oral steroids, she will require intravenous hydrocortisone to cover the physiological stress of labor and delivery. Ideal regimes are uncertain: many practitioners prescribe $50-100 \mathrm{mg}$ intravenous hydrocortisone 8 hourly (eg, $50 \mathrm{mg}$ tds if taking 7.5 to $<20 \mathrm{mg}$ prednisolone; $100 \mathrm{mg}$ tds if taking $\geq 20 \mathrm{mg}$ prednisolone).

Women receiving standard prophylactic (once daily) doses of LMWH should discontinue it at the onset of spontaneous labor, or the night before induced labor or elective cesarean section. They will be able to have regional anesthesia (epidural or spinal) 12 hours after the last LMWH dose. Women receiving high-dose prophylactic or therapeutic (twice-daily) LMWH should discontinue it at the onset of spontaneous labor or 24 hours before induced labor or elective cesarean section. They will be able to have regional anesthesia 24 hours after the last LMWH dose. Alternative analgesia options, such as a patient-controlled analgesia opiate pump, are available for patients requesting additional pain relief who are not within a safe post-LMWH time frame for regional anesthesia. LMWH can be recommenced 4 hours after a spinal injection or after an epidural catheter has been removed, provided the woman is hemodynamically stable. The epidural catheter should be removed $>12$ hours after the most recent LMWH injection. ${ }^{18}$

\section{Postpartum challenge: flare, other risks, and follow-up}

The puerperium (weeks 0-6 postpartum) was thought to be a period of high risk of lupus flares, ${ }^{10}$ although some groups have found that disease activity decreases after pregnancy. ${ }^{45}$ Of seven prospective comparative studies assessing pregnancy and postpartum flares, three showed an increased risk of flares; four did not. ${ }^{79}$ Women taking HCQ, azathioprine, and/or steroids for immunosuppression in pregnancy should be reassured that these medications are safe for breastfeeding, and advised to continue.

If a woman is taking long-term oral steroids, she should have received intravenous hydrocortisone to cover the physiological stress of labor and delivery. Postpartum, doubling her usual dose of prednisolone for 2-3 days would be considered, particularly if she was unwell or had a prolonged labor or complicated delivery, and then her usual dose would be continued.

A member of the multidisciplinary lupus team should review the woman on the ward prior to her discharge, with a follow-up outpatient appointment for 4-6 weeks postpartum, depending on antenatal or intrapartum lupus complications and risk factors for lupus complications. This review is to detect and manage any postpartum lupus complications, and ensure a smooth return to rheumatology outpatient services for ongoing care. A thorough history, physical examination, blood pressure, and urinalysis should be undertaken, with assessment of FBC, U\&E, creatinine, LFTs, and disease activity markers (erythrocyte sedimentation rate, C-reactive protein, anti-dsDNA, and complement).

The woman's obstetric history should also be considered. A recent study of 3,977 parous women with SLE over a 
38-year period in Sweden showed that those with adverse pregnancy outcomes related to maternal-placental syndrome (hypertensive disorders of pregnancy, SGA, placental abruption, and/or stillbirth) have an increased risk (hazard ratio 1.64) and accelerated development of cardiovascular events (coronary artery disease, stroke, peripheral vascular disease, and/or death from cardiovascular causes) in later life when compared with women who had uncomplicated pregnancies. The risk was doubled in women with SLE and maternal-placental syndrome who delivered a baby at $<34$ weeks of gestation. ${ }^{80}$

The puerperium is also a period of increased VTE risk. All women who received antenatal LMWH should continue this for 6 weeks postpartum, in a once-daily dosage (recent weight). All women should undergo a postpartum VTE risk assessment: many may require a 10-day course for obstetric or other reasons (risk factors include age, BMI, parity, aPL, cesarean section, postpartum hemorrhage); the threshold for postpartum prophylaxis is lower than antenatal as it is a time of greater risk. ${ }^{18}$ Women with previous or recent VTE may require long-term anticoagulation: LMWH can be switched to warfarin when the risk of hemorrhage has reduced, usually at days 5-7 postpartum, with continuation of LMWH until the international normalized ratio reaches therapeutic range. Some women may be accustomed to LMWH use during pregnancy and may prefer to continue postpartum, even for an extended course, rather than switching to warfarin with its monitoring blood tests.

\section{Postpartum challenge: contraception}

In addition to rheumatology and/or obstetric medicine follow-up appointments, the woman should also attend her routine 6-week mother-and-baby check at her general practitioner clinic. This includes clinical review, contraception discussion, cervical smear appointment (if overdue), and 6-week baby check.

Postpartum counseling and provision of contraception is particularly important in patients with SLE (and all patients with chronic medical conditions) as planned pregnancy is associated with fewer complications and higher pregnancy success rates. ${ }^{10}$

Barrier methods (condom, female condom, diaphragm, cap, sponge) are convenient and readily available but have a $15 \%-32 \%$ failure rate with typical use. Condoms and female condoms also protect against sexually transmitted infections. ${ }^{81}$

Hormonal methods can be divided into those containing estrogen and progestogen (combined oral contraceptive pill
[COCP], contraceptive patch, and contraceptive ring), and progestogen-only methods (progestogen-only pill, implant, Depo-Provera injection, and Mirena intrauterine system [IUS]).

Estrogen-containing contraceptives increase a woman's VTE risk, so they are contraindicated in women with aPL or APS, moderate-severe active SLE (including lupus nephritis), hypertension, smoking, obesity, or previous VTE. ${ }^{82}$ Women with well-defined SLE with stable and/or low-active disease may be suitable to use the COCP if they wish: ${ }^{82} \mathrm{a}$ systematic review of 14 studies, including two randomized controlled trials, showed that combined oral contraceptive use does not lead to increased disease flares or worsening disease activity in women with inactive or stable SLE. ${ }^{83,84}$

Depo-Provera, progestogen subdermal implants, and the IUS are long-acting reversible contraception methods which are reliable (failure rate $>1 \%$ ), less reliant on patient compliance, and last for up to 3 months, 3 years, and 5 years, respectively. The IUS also reduces menstrual blood loss. Progestogen-only pills are effective if taken correctly but have a narrower compliance window than COCPs. Progestogenonly methods are safe for women in whom estrogens are contraindicated, including those with VTE and hypertension, as they do not increase VTE risk. They may cause a small, reversible reduction in bone density. ${ }^{81,82,85}$

The intrauterine contraceptive device, or copper-containing coil, is also an extremely effective method of contraception, and does not carry additional VTE risks. It can increase menstrual bleeding, so it may not be suitable for women on long-term warfarin or $\mathrm{LMWH}^{81,86}$

\section{Conclusion}

Women with SLE, particularly those with associated lupus nephritis or APS, are at increased risk of adverse pregnancy outcomes. Care should be coordinated by obstetricians, midwives, and rheumatologists with expertise in the care of high-risk pregnancies. Maintaining disease remission and treating any flares rapidly is vital. All clinicians involved in the management of SLE pregnancies should feel comfortable counseling women regarding medication use in pregnancy and in particular advise women not to discontinue antirheumatic drugs compatible with pregnancy. Evidence-based guidelines from the UK and Europe are now available.

\section{Disclosure}

The authors report no conflicts of interest in this work.

\section{References}

1. Rees F, Doherty M, Grainge M, Davenport G, Lanyon P, Zhang W. The incidence and prevalence of systemic lupus erythematosus in the UK, 1999-2012. Ann Rheum Dis. 2016;75(1):136-141. 
2. Rahman A, Isenberg D. Systemic lupus erythematosus. $N$ Engl J Med. 2008;358(9):929-939.

3. Dall'Era M. Chapter 21. Systemic lupus erythematosus. In: Imboden JB, Stone JH, Hellmann DB, eds. Current rheumatology diagnosis \& treatment. 3 ed. USA: McGraw-Hill; 2013.

4. Petri M. Epidemiology of systemic lupus erythematosus. Best Pract Res Clin Rheumatol. 2002;16(5):847-858.

5. Gladman D, Urowitz M. Chapter 69. Prognosis, Mortality, and Morbidity in Systemic Lupus Erythematosus. In: Wallace D, Hahn B, eds. Dubois Lupus Erythematosus. 7th ed. Philadelphia: Lippincott Williams \& Wilkins; 2007:1333-1353.

6. Hahn B, McMahon M, Wilkinson A, et al. American College of Rheumatology guidelines for screening, treatment, and management of lupus nephritis. Arthritis Care Res (Hoboken). 2012;64(6):797-808.

7. Ruiz-Irastorza $\mathrm{G}$, Khamashta $\mathrm{M}$. Lupus and pregnancy: integrating clues from the bench and bedside. Eur J Clin Invest. 2011;41(6):672-678.

8. Ateka-Barrutia O, Nelson-Piercy C. Management of rheumatologic diseases in pregnancy. International Journal of Clinical Rheumatology. 2012;7(5):541-558.

9. Ostensen M, Cetin I. Autoimmune connective tissue diseases. Best Pract Res Clin Obstet Gynaecol. 2015;29(5):658-670.

10. Ateka-Barrutia O, Nelson-Piercy C. Connective tissue disease in pregnancy. Clin Med. 2013;13(6):580-584.

11. Bellver J, Pellicer A. Ovarian stimulation for ovulation induction and in vitro fertilization in patients with systemic lupus erythematosus and antiphospholipid syndrome. Fertil Steril. 2009;92(6):1803-1810.

12. National Institute for Health and Care Excellence :NICE). Hypertension in pregnancy: diagnosis and management. [CG107]. NICE clinical guideline 107. Manchester: National Institute for Health and Care Excellence; 2011.

13. Chappell L, Duckworth S, Seed P, et al. Diagnostic accuracy of placental growth factor in women with suspected preeclampsia: a prospective multicenter study. Circulation. 2013;128(19):2121-2131.

14. Doria A, Tincani A, Lockshin M. Challenges of lupus pregnancies. Rheumatology (Oxford). 2008;47(Suppl 3):iii9-12.

15. Clowse M, Magder L, Witter F, Petri M. The impact of increased lupus activity on obstetric outcomes. Arthritis Rheum. 2005;52(2):514-521.

16. Miyakis S, Lockshin M, Atsumi T, et al. International consensus statement on an update of the classification criteria for definite antiphospholipid syndrome (APS). J Thromb Haemost. 2006;4(2):295-306.

17. Soh M, Pasupathy D, Gray G, Nelson-Piercy C. Persistent antiphospholipid antibodies do not contribute to adverse pregnancy outcomes. Rheumatology (Oxford). 2013;52(9):1642-1647.

18. Royal College of Obstetricians and Gynaecologists (RCOG). Reducing the risk of venous thromboembolism during pregnancy and the puerperium. Green-top Guideline No. 37a London: RCOG; 2015.

19. Nelson-Piercy C. Handbook of Obstetric Medicine. 5th ed. Florida, USA: CRC Press; 2015.

20. Clowse M, Jamison M, Myers E, James A. A national study of the complications of lupus in pregnancy. Am J Obstet Gynecol. 2008;199(2):127. e121-e126.

21. Andrade R, Sanchez M, Alarcon G, et al. Adverse pregnancy outcomes in women with systemic lupus erythematosus from a multiethnic US cohort: LUMINA (LVI) [corrected]. Clin Exp Rheumatol. 2008, 26(2):268-274.

22. Singh A, Chowdhary V. Pregnancy-related issues in women with systemic lupus erythematosus. Int J Rheum Dis. 2015;18(2):172-181.

23. Imbasciati E, Tincani A, Gregorini G, et al. Pregnancy in women with pre-existing lupus nephritis: predictors of fetal and maternal outcome. Nephrol Dial Transplant. 2009;24(2):519-525.

24. Germain S, Nelson-Piercy C. Lupus nephritis and renal disease in pregnancy. Lupus. 2006;15(3):148-155.

25. Askie LM, Duley L, Henderson-Smart DJ, Stewart LA. Antiplatelet agents for prevention of pre-eclampsia: a meta-analysis of individual patient data. The Lancet. 2007;369(9575):1791-1798.

26. National Institute for Health and Care Excellence (NICE). Antenatal care for uncomplicated pregnancies. [CG62]. NICE clinical guideline 62. Manchester: National Institute for Health and Care Excellence; 2016.
27. Royal College of Obstetricians and Gynaecologists (RCOG). Healthy eating and vitamin supplements in pregnancy. Patient information leaflet. London: RCOG; 2014.

28. Hofmeyr G, Lawrie T, Atallah A, Duley L, Torloni M. Calcium supplementation during pregnancy for preventing hypertensive disorders and related problems. Cochrane Database Syst Rev. 2014;6:CD001059.

29. Schwarz E, Manzi S. Risk of unintended pregnancy among women with systemic lupus erythematosus. Arthritis Rheum. 2008;59(6):863-866.

30. Gotestam Skorpen C, Hoeltzenbein M, Tincani A, et al. The EULAR points to consider for use of antirheumatic drugs before pregnancy, and during pregnancy and lactation. Ann Rheum Dis. 2016;75(5): 795-810.

31. Flint J, Panchal S, Hurrell A, et al. BSR and BHPR guideline on prescribing drugs in pregnancy and breastfeeding-Part I: standard and biologic disease modifying anti-rheumatic drugs and corticosteroids. Rheumatology (Oxford). Epub 2016 Jan 10.

32. Flint J, Panchal S, Hurrell A, et al. BSR and BHPR guideline on prescribing drugs in pregnancy and breastfeeding-Part II: analgesics and other drugs used in rheumatology practice. Rheumatology (Oxford). Epub 2016 Jan 10.

33. Ostensen M, Khamashta M, Lockshin M, et al. Anti-inflammatory and immunosuppressive drugs and reproduction. Arthritis Res Ther. 2006;8(3):209.

34. Cauldwell M, Nelson-Piercy C. Maternal and fetal complications of systemic lupus erythematosus. The Obstetrician \& Gynaecologist. 2012;14(3):167-174.

35. British National Formulary (BNF) 71. British Medical Association, and Royal Pharmaceutical Society, London: March 2016

36. Silva C, Bonfa E, Ostensen M. Maintenance of fertility in patients with rheumatic diseases needing antiinflammatory and immunosuppressive drugs. Arthritis Care Res (Hoboken). 2010;62(12):1682-1690.

37. Li D, Liu L, Odouli R. Exposure to non-steroidal anti-inflammatory drugs during pregnancy and risk of miscarriage: population based cohort study. BMJ. 2003;327(7411):368.

38. Clowse M, Magder L, Witter F, Petri M. Hydroxychloroquine in lupus pregnancy. Arthritis Rheum. 2006;54(11):3640-3647.

39. Izmirly P, Costedoat-Chalumeau N, Pisoni C, et al. Maternal use of hydroxychloroquine is associated with a reduced risk of recurrent antiSSA/Ro-antibody-associated cardiac manifestations of neonatal lupus. Circulation. 2012;126(1):76-82.

40. Sau A, Clarke S, Bass J, Kaiser A, Marinaki A, Nelson-Piercy C. Azathioprine and breastfeeding: is it safe? BJOG. 2007;114(4):498-501.

41. Webster P, Wardle A, Bramham K, Webster L, Nelson-Piercy C, Lightstone L. Tacrolimus is an effective treatment for lupus nephritis in pregnancy. Lupus. 2014;23(11):1192-1196.

42. UK Teratology Information Service. Use of mycophenolate mofetil in pregnancy (v2.1). 2016. http://www.medicinesinpregnancy.org/ bumps/monographs/USE-OF-MYCOPHENOLATE-MOFETIL-INPREGNANCY/. Accessed June 9, 2016.

43. UK Teratology Information Service. Use of methotrexate in pregnancy (v1). 2016. http://www.medicinesinpregnancy.org/bumps/monographs/ USE-OF-METHOTREXATE-IN-PREGNANCY/. Accessed June 9, 2016.

44. Petri M. The Hopkins Lupus Pregnancy Center: ten key issues in management. Rheum Dis Clin North Am. 2007;33(2):227-235, v.

45. Andrade R, McGwin GJ, Alarcon G, et al. Predictors of post-partum damage accrual in systemic lupus erythematosus: data from LUMINA, a multiethnic US cohort (XXXVIII). Rheumatology (Oxford). 2006;45(11):1380-1384.

46. Ruiz-Irastorza G, Ramos-Casals M, Brito-Zeron P, Khamashta M. Clinical efficacy and side effects of antimalarials in systemic lupus erythematosus: a systematic review. Ann Rheum Dis. 2010;69(1):20-28.

47. Levy R, Vilela V, Cataldo M, et al. Hydroxychloroquine (HCQ) in lupus pregnancy: double-blind and placebo-controlled study. Lupus. 2001;10(6): 401-404.

48. Abbassi-Ghanavati M, Greer LG, Cunningham FG. Pregnancy and laboratory studies: a reference table for clinicians. Obstetrics \& Gynecology. 2009;114(6):1326-1331. 
49. Buyon JP, Kalunian KC, Ramsey-Goldman R, et al. Assessing disease activity in SLE patients during pregnancy. Lupus. 1999;8(8):677.

50. Hall M, Brunskill N. Renal disease in pregnancy. Obstetrics, Gynaecology and Reproductive Medicine. 2012;23(2):31-37.

51. Bramham K, Nelson-Piercy C. Chapter 295: Pregnancy in patients with chronic kidney disease and on dialysis. In: Turner N, Lameire N, Goldsmith D, Winearls C, Himmelfarb J, Remuzzi G, eds. Oxford Textbook of Clinical Nephrology. 4th ed. Oxford, UK: Oxford University Press; 2016:2449-2555.

52. Beca S, Rodriguez-Pinto I, Alba M, Cervera R, Espinosa G. Development and validation of a risk calculator to differentiate flares from infections in systemic lupus erythematosus patients with fever. Autoimmun Rev. 2015;14(7):586-593.

53. Milne F, Redman C, Walker J, et al. The pre-eclampsia community guideline (PRECOG): how to screen for and detect onset of pre-eclampsia in the community. BMJ. 2005;330(7491):576-580.

54. Smyth A, Oliveira G, Lahr B, Bailey K, Norby S, Garovic V. A systematic review and meta-analysis of pregnancy outcomes in patients with systemic lupus erythematosus and lupus nephritis. Clin J Am Soc Nephrol. 2010;5(11):2060-2068.

55. de Jesus G, de Jesus N, Levy R, Klumb E. The use of angiogenic and antiangiogenic factors in the differential diagnosis of pre-eclampsia, antiphospholipid syndrome nephropathy and lupus nephritis. Lupus. 2014;23(12):1299-1301.

56. Bramham K, Seed P, Lightstone L, et al. Diagnostic and predictive biomarkers for pre-eclampsia in patients with established hypertension and chronic kidney disease. Kidney Int. 2016;89(4):874-885.

57. Yan Yuen S, Krizova A, Ouimet JM, Pope JE. Pregnancy outcome in systemic lupus erythematosus (SLE) is improving: results from a case control study and literature review. The open rheumatology journal. 2008;2(1).

58. Rahman F, Rahman J, Al-Suleiman S, Rahman M. Pregnancy outcome in lupus nephropathy. Arch Gynecol Obstet. 2005;271(3):222-226.

59. Dhar J, Essenmacher L, Ager J, Sokol R. Pregnancy outcomes before and after a diagnosis of systemic lupus erythematosus. Am J Obstet Gynecol. 2005;193(4): 1444-1455.

60. Clark C, Spitzer K, Laskin C. Decrease in pregnancy loss rates in patients with systemic lupus erythematosus over a 40-year period. J Rheumatol. 2005;32(9):1709-1712.

61. Gladman D, Tandon A, Ibanez D, Urowitz M. The effect of lupus nephritis on pregnancy outcome and fetal and maternal complications. J Rheumatol. 2010;37(4):754-758.

62. Ehrenstein M, Isenberg D. 6.6.1 Systemic lupus erythematosus in adults - clinical features and aetiopathogenesis. In: Isenberg D, Maddison P, Woo P, Glass D, Breedveld F, eds. Oxford Textbook of Rheumatology. 3rd ed. Oxford: Oxford University Press; 2004:819-841.

63. Boh E. Neonatal lupus erythematosus. Clin Dermatol. 2004;22(2): $125-128$.

64. Izmirly P, Llanos C, Lee L, Askanase A, Kim M, Buyon J. Cutaneous manifestations of neonatal lupus and risk of subsequent congenital heart block. Arthritis Rheum. 2010;62(4):1153-1157.

65. Brucato A, Frassi M, Franceschini F, et al. Risk of congenital complete heart block in newborns of mothers with anti-Ro/SSA antibodies detected by counterimmunoelectrophoresis: a prospective study of 100 women. Arthritis Rheum. 2001;44(8):1832-1835.

66. Lee L. The clinical spectrum of neonatal lupus. Arch Dermatol Res. 2009;301(1):107-110.

67. Brito-Zeron P, Izmirly P, Ramos-Casals M, Buyon J, Khamashta M. Autoimmune congenital heart block: complex and unusual situations. Lupus. 2016;25(2):116-128.
68. Levesque K, Morel N, Maltret A, et al. Description of 214 cases of autoimmune congenital heart block: Results of the French neonatal lupus syndrome. Autoimmun Rev. 2015;14(12):1154-1160.

69. Friedman D, Duncanson L, Glickstein J, Buyon J. A review of congenital heart block. Images Paediatr Cardiol. 2003;5(3):36-48.

70. Buyon J, Hiebert R, Copel J, et al. Autoimmune-associated congenital heart block: demographics, mortality, morbidity and recurrence rates obtained from a national neonatal lupus registry. JAm Coll Cardiol. 1998; 31(7):1658-1666.

71. Brucato A, Cimaz R, Caporali R, Ramoni V, Buyon J. Pregnancy outcomes in patients with autoimmune diseases and anti-Ro/SSA antibodies. Clin Rev Allergy Immunol. 2011;40(1):27-41.

72. Jaeggi E, Hamilton R, Silverman E, Zamora S, Hornberger L. Outcome of children with fetal, neonatal or childhood diagnosis of isolated congenital atrioventricular block. A single institution's experience of 30 years. J Am Coll Cardiol. 2002;39(1):130-137.

73. Friedman D, Llanos C, Izmirly P, et al. Evaluation of fetuses in a study of intravenous immunoglobulin as preventive therapy for congenital heart block: Results of a multicenter, prospective, open-label clinical trial. Arthritis Rheum. 2010;62(4):1138-1146.

74. Pisoni C, Brucato A, Ruffatti A, et al. Failure of intravenous immunoglobulin to prevent congenital heart block: Findings of a multicenter, prospective, observational study. Arthritis Rheum. 2010;62(4):1147-1152.

75. Cnossen J, Morris R, ter Riet G, et al. Use of uterine artery Doppler ultrasonography to predict pre-eclampsia and intrauterine growth restriction: a systematic review and bivariable meta-analysis. CMAJ. 2008; 178(6):701-711.

76. Izmirly P, Rivera T, Buyon J. Neonatal lupus syndromes. Rheum Dis Clin North Am. 2007;33(2):267-285, vi.

77. Royal College of Obstetricians and Gynaecologists (RCOG). Antenatal corticosteroids to reduce neonatal morbidity and mortality. Green-top Guideline No. 7. London: RCOG; 2010.

78. Royal College of Obstetricians and Gynaecologists (RCOG). Magnesium sulphate to prevent cerebral palsy following preterm birth. London RCOG: Scientific Impact Paper No. 29; 2011.

79. Baer A, Witter F, Petri M. Lupus and pregnancy. Obstet Gynecol Surv. 2011;66(10):639-653.

80. Soh M, Dib F, Nelson-Piercy C, Westgren M, McCowan L, Pasupathy D. Maternal-placental syndrome and future risk of accelerated cardiovascular events in Parous Swedish women with systemic lupus erythematosus - a population-based retrospective cohort study with time-to-event analysis. Rheumatology (Oxford). 2016;55(7):1235-1242.

81. Wiles K, Nelson-Piercy C. Chapter 293: Contraception in patients with kidney disease. In: Turner N, Lameire N, Goldsmith D, Winearls C, Himmelfarb J, Remuzzi G, eds. Oxford Textbook of Clinical Nephrology. 4th ed. Oxford, UK: Oxford University Press; 2016:2530-2536.

82. FSRH (Faculty of Sexual and Reproductive Healthcare). UK Medical Eligibility Criteria (UKMEC) for contraceptive use. London FSRH; 2016.

83. Culwell K, Curtis K, del Carmen Cravioto M. Safety of contraceptive method use among women with systemic lupus erythematosus: a systematic review. Obstet Gynecol. 2009;114(2 Pt 1):341-353.

84. Petri M, Kim M, Kalunian K, et al. Combined oral contraceptives in women with systemic lupus erythematosus. NEngl J Med. 2005;353(24): 2550-2558.

85. Chabbert-Buffet N, Amoura Z, Scarabin P, et al. Pregnane progestin contraception in systemic lupus erythematosus: a longitudinal study of 187 patients. Contraception. 2011;83(3):229-237.

86. Clowse M. Managing contraception and pregnancy in the rheumatologic diseases. Best Pract Res Clin Rheumatol. 2010;24(3):373-385. 
Open Access Rheumatology: Research and Reviews is an international, peerreviewed, open access journal publishing original research, reports, editorials, reviews and commentaries on all aspects of clinical and experimental rheumatology in the clinic and laboratory including the following topics: Pathology, pathophysiology of rheumatological diseases; Investigation, treatment and management of rheumatological diseases; Clinical trials and novel pharmacological approaches for the treatment of rheumatological disorders. The manuscript management system is completely online and includes a very quick and fair peer-review system, which is all easy to use. Visit http://www.dovepress.com/ testimonials.php to read real quotes from published authors. 